\title{
European Aligner Society Autumn Meeting
}

The European Aligner Society (EAS) recently held its 2020 Autumn Meeting to continue to explore the perpetual advances in technologies and techniques in aligner orthodontics. The meeting, which took a virtual format to meet the needs of these challenging times, was moderated by EAS President Alain Souchet and Scientific Director Tommaso Castroflorio. It comprised three plenary sessions with speakers presenting the latest findings and experiences of aligner therapy.

As befitting a European organisation, the lectures were delivered by an international line-up of expert speakers, and attracted 207 delegates from around the world. In the space of a day, delegates were taken on a world tour, taking in the latest orthodontic expertise.

The global excursion started in Greece with Dr Gina Theodoridis whose lecture examined the factors and biomechanical concepts which affect the effectiveness of distalising the posterior teeth with aligners, and explored the realistic outcomes which can be achieved in such circumstances.

Dr John Kaku from Japan is an Instructor at the Japanese Academy of Non-Extraction Orthodontics. For the EAS meeting, Dr Kaku took delegates on a tour of his practice, demonstrating the whole patient journey and highlighting how involving a well-trained team and the right digital tools contributed to the success of the practice.

The Vice-President of the Portuguese Aligner Society (SPAD) Dr Pedro Monteiro expanded on the changing orthodontic landscape and the behavioural shift amongst consumers. He highlighted the importance of reinventing the patient journey using artificial intelligence and teledentistry in the virtual era and the need for building a virtual practice for what is not just the post-COVID era, but the new reality.

Dr Rafi Romano delivered his lecture from his orthodontic practice in Tel Aviv, Israel. His emphasis on adult and aesthetic orthodontics using state-of-the-art technologies was the focus of his session. His lecture focused on chairside tools which help orthodontists visualise, discuss and predict treatment options before beginning treatment and, later on, to assess the progress and fine-tune the treatment to maximise efficiency, shorten back-and-forth and the need for additional aligners.

Dr Dalia Latkauskiene from Lithuania presented on the topic of asymmetries in aligner treatment. If not properly diagnosed, slight asymmetries tend to be revealed throughout orthodontic treatment, thereby extending treatment time and hindering final outcomes. Dr Latkauskiene presented different plans of aligner treatment for dentoalveolar and skeletal asymmetries and advised her peers to be cautious with asymmetries of skeletal growth when treating children.

John Morton from the USA discussed the Invisalign SmartForce features, SmartTrack material and SmartStage technology which together provide the optimised progression of tooth movements, reduce unwanted interferences and increase the predictability of Invisalign treatment.

Dr Sandra Tai from Canada's presentation looked at the results of a research study completed at the University of British Columbia comparing skeletal, dental and soft tissue effects of Class II malocclusions treated with Invisalign with mandibular advancement compared with twin block functional appliances.

Dr Tomas Salazar from Chile presented 'In office aligner workflow' underlining that clear aligners have their place in an orthodontic clinical practice. He showcased the importance of the digital workflow in the treatment of malocclusions as it gives the exact information of how much and when each tooth will move.

For those who would like to watch the lectures in their own time it is possible to do so by visiting www.eas-aligners.com.

\section{New e-learning programme supports return to training}

A new programme designed to support medical and dental trainees returning to training after prolonged absence is now available on Health Education England's

e-Learning for Healthcare Hub.

Supported Return to Training for

Trainees has been developed to improve the return to training experience for all medical and dental trainees who have been out of training for three months or more.

This e-learning programme aims to:

- Help trainees understand the supported return to training process

- Recognise and discuss common concerns for returning trainees
- Hear the positive experiences of other trainees' return to training.

The programme is aimed at trainees planning an absence from training, and those planning their return to training.

The course, which is formative and not assessed, takes approximately 30 to 60 minutes to complete and comprises the following topics:

- Introduction

- Your (trainee) concerns

- The SuppoRTT process

- Keep in touch/shared parental leave in touch/return to training days

- People who can support you
- Your wellbeing

- Summary

- Links to HEE SuppoRTT teams' webpages.

It was developed by Health Education England North West (HEE NW), in conjunction with the blended learning team at Lancashire Teaching Hospitals NHS Foundation Trust.

For more information and to access the course, please visit the Supported Return to Training for Trainees programme page at https://www.e-lf.org.uk/programmes/ supported-return-to-training-for-trainees/. 